\title{
Development and validation of a symptom questionnaire for recording outcomes in adult lacrimal surgery*
}

\author{
N. Mistry ${ }^{1}$, T.J. Rockley ${ }^{1}$, T. Reynolds ${ }^{2}$, C. Hopkins ${ }^{3}$ \\ Department of Otorhinolaryngology, Queen's Hospital, Burton upon Trent, Staffordshire, United Kingdom \\ 2 Department of Chemical Pathology, Queen's Hospital, Burton upon Trent, Staffordshire, United Kingdom \\ Department of Otorhinolaryngology, Guy's and St Thomas' Hospital, London, United Kingdom
}

SUMMARY Background: A symptom-based questionnaire (the 'Lac-Q' questionnaire) for adult patients undergoing lacrimal drainage surgery was developed. The questionnaire yields a numerical score that can be used to assess severity of symptoms.

Methodology: In this study, the questionnaire was evaluated in 17 consecutive patients undergoing 22 dacryocystorhinostomy (DCR) procedures. The questionnaire was administered pre-and postoperatively. The pathology encountered at operation was recorded. The success of surgery was judged by patient satisfaction, endoscopic evaluation of DCR stomal patency, and objective lacrimal drainage testing using the functional endoscopic dye test (FEDT). In a further group of 12 pre-operative cases, the questionnaire was repeated after 4-6 weeks but before surgery, to assess test-retest reliability in the absence of clinical change.

Results: The Lac-Q questionnaire was based on two broad categories of eye-specific scores and social impact scores. A numerical score, the 'Lac- $Q$ ' score, was generated pre- and postoperatively. When compared to pre-operative scores, the reduction in Lac- $Q$ scores postoperatively was significant. Postoperative scores also correlated well with objective lacrimal drainage testing using the FEDT. Analysis of symptom scores shows that the questionnaire was reliable with regard to content validity, internal consistency, test-retest reliability, and responsiveness to clinical change.

Conclusions: We conclude that the Lac-Q questionnaire is a useful clinical tool to evaluate outcomes after adult lacrimal surgery.

Key words: patient-related outcome measure (PROM), lacrimal surgery outcome, patient symptom questionnaire, dacryocystorhinostomy (DCR), lacrimal duct surgery

\section{INTRODUCTION}

The use of patient-rated outcome measures (PROMs) is increasingly a feature of modern surgical practice ${ }^{(1,2)}$. Patientcentred methods of measuring surgical outcomes, in terms of both symptoms and social impact of disease, have been promoted in recent years to develop standards for surgical practice and enable patients to make better informed choices about their health care.

The dacryocystorhinostomy (DCR) operation for lacrimal drainage is now a common procedure in rhinological practice.
There are standardised questionnaires available for recording outcomes for several other rhinological procedures ${ }^{(3)}$, but at present there is no generally accepted symptom-based method for recording outcomes of lacrimal surgery. There are several reasons why this is so. Firstly, nasolacrimal duct obstruction (NDO) can give rise to a variety of pathologies apart from simple tear drainage obstruction; dacryocoele, mucocoele, dacryocystitis, calculus or fistula (4). Patients may therefore present with any of the following four symptoms, in varying severity; epiphora, mucous discharge, pain, or medial orbital swelling. 
Secondly, lacrimal duct obstruction may cause no symptoms at all in some patients, especially those who are elderly and who have reduced tear production. The sac itself can absorb a certain volume of tears and this can balance the reduced tear production, such that epiphora does not occur despite complete distal duct blockage ${ }^{(5)}$. Alternatively, symptoms may be present, but not sufficiently severe to be perceived as a health problem, and therefore the patient may not seek medical attention. Thirdly, there is no pre-operative clinical test that can predict severity of symptoms, so there is no objective 'gold standard' against which symptoms can be judged.

The assessment of surgical success or failure is also problematic. The formation of a technically satisfactory DCR stoma may not necessarily resolve symptoms, as there can be additional pathology within the upper lacrimal canaliculi causing obstruction. Simple observation of stomal patency is thus not sufficient to judge operative success. There is however one objective clinical postoperative test that can reliably predict surgical success after DCR. This is the functional endoscopic dye test (FEDT). In this test, a fluorescein dye eyedrop is instilled in the ipsilateral conjunctiva and the DCR stoma then observed intranasally using an endoscope ${ }^{(6)}$. If dye is seen to appear in the stoma, lacrimal drainage is demonstrably present; appearance of dye within two minutes has been shown to correlate with patient satisfaction following surgery ${ }^{(7)}$.

Despite these difficulties, there have been several studies that have evaluated outcomes after DCR surgery. However, these studies have had limitations in terms of using non-validated questionnaires to assess symptoms, or restricting the questions such that some symptoms were not included ${ }^{(8-10)}$. Many also used generic quality of life studies ${ }^{(7,11)}$, which have limited applicability to lacrimal disease, and some only evaluated patients after surgery, lacking pre-operative comparison ${ }^{(12)}$.

The purpose of this study, therefore, was to develop and validate a symptom-based questionnaire for adult patients undergoing lacrimal surgery. From the foregoing discussion, it may be surmised that an ideal questionnaire would have the following characteristics. It would be easy to administer and have a simple clinical utility for both assessing patients pre-operatively and judging success or failure postoperatively. It would be able to quantify the severity of each of the four symptoms of lacrimal obstruction; epiphora, sticky eye, pain, and swelling. It would also record and quantify the overall quality of life and social impact issues that lacrimal patients experience.

\section{MATERIALS AND METHODS}

\section{Development of the Lac-Q questionnaire}

The presenting symptomatology and pathology of 100 consecutive patients referred for treatment of lacrimal obstruction was reviewed. This was to determine the range and severity of symptoms they experienced. It was also to assess the social impact of these symptoms i.e. the effect that their symptoms had on day-to-day living, medical attendance, social embarrassment, and disability. As a result of this analysis, the Lac-Q questionnaire was developed (see Appendix for a copy of the questionnaire). The numerical score of the questionnaire for each patient is the sum of three scores, representing three separate domains: a symptom score for the right eye; a symptom score for the left eye; and a score for social impact.

\section{Clinical testing of the Lac-Q questionnaire}

1. Use of the questionnaire to assess severity of symptoms and outcomes of surgery.

The Lac-Q questionnaire was administered both pre-operatively and postoperatively to 17 consecutive adult patients attending for endonasal DCR surgery. The study had local ethical and institutional research body approval. Pre-operative administration of the questionnaire was done in outpatient clinic at the time of listing for surgery. Postoperative evaluation was carried out in clinic 8 - 10 weeks after surgery. If lacrimal stenting tubes had been inserted at operation, the postoperative evaluation was carried out eight weeks after the tubes were removed. The questionnaire was re-administered and a record was made of patient satisfaction with the surgical outcome. If the patient said that their symptoms had improved sufficiently for them not to wish for any further treatment, the patient was deemed to be 'satisfied' with the operation. If they said that their eye was still causing sufficient problems to wish for further treatment, the patient was deemed to be 'not satisfied' with the procedure. Clinical assessment of the surgical outcome was then performed. The DCR stoma in the lateral nasal wall was inspected with a nasoendoscope and categorised as 'patent' or 'stenosed.' A FEDT test was then carried out. A single fluorescein eye drop was then instilled in the ipsilateral conjunctiva. The patient was asked to blink normally. The stoma was then re-inspected at one-minute intervals and the time until fluorescein appearance in the stoma noted. The interval was recorded as $<2$ minutes, 2 - 5 minutes, or not at all (i.e. inspection showed no dye even after 10 minutes) (Figure 1).

For each patient, the pathology encountered at operation was categorised as being in one of the following three categories, in increasing severity: simple obstruction; obstruction with mucocoele; obstruction (with or without mucocoele) with superadded infection.

\section{Test-retest reliability of the questionnaire.}

In a separate group of 12 patients awaiting endonasal DCR surgery, the Lac-Q questionnaire was re-administered after 4-8 weeks, to assess test-retest reliability of the responses in the absence of surgical intervention.

\section{Data Analysis and validation of questionnaire}

The Lac-Q questionnaire was analysed in terms of its reliability, validity, responsiveness and interpretability. 


\section{Reliability}

Internal consistency was assessed by analysing correlation between the eye symptom scores and the social impact scores for each patient, both pre- and post-operatively. If these test items within the questionnaire were shown to be intercorrelated, it would indicate that the symptoms recorded from the eye were indeed the problems that were impacting adversely on the patient's lifestyle. Spearman's rank correlation coefficient was therefore used to demonstrate correlation and homogeneity between items within the scale. The stability and reproducibility of the scores was evaluated by comparing the two sets of pre-operative scores, taken from questionnaires administered 4 - 8 weeks apart, in the separate group of 12 preoperative patients. A test-retest reliability coefficient of $\geq 0.70$ was required to show evidence of correlation between matched responses.

\section{Validity}

Validity was evaluated in several ways. Pre-operative scores were compared with postoperative scores according to the patient outcome, to allow assessment of the discriminatory value of the questionnaire at identifying 'healthy' vs. 'unhealthy' patients (discriminative validity). Assessment of correlation between pre-operative scores and severity of pathology encountered at operation was also undertaken, to give an indication of construct validity.

\section{Responsiveness}

Responsiveness was assessed by measuring the change in scores following surgery. The relationship between post-operative scores and operative success (or failure) as evidenced by patient satisfaction, stomal patency and FEDT results was also analysed.

\section{Interpretability}

Clinical interpretability was assessed by comparing the scores between symptomatic and non-symptomatic patients. The minimally important difference (minimal change in score, which means operation has been successful) was noted.

\section{Statistics}

The statistical significance of the validity and responsiveness tests was determined using the Wilcoxon-signed ranks test and tests of correlation (Kendall's Tau-b and Spearman's rank).

\section{RESULTS}

\section{Format of the questionnaire (see Appendix)}

From the clinical case records analysed, there were found to be essentially two broad categories of symptoms. Firstly, there were varying degrees of eye-specific symptoms: tearing, stickiness, pain or swelling. Secondly, there were symptoms that reflected the overall social impact of the disease on the patient. This was evidenced by the need to attend medical clinics, social embarrassment, blurring of vision, and the effect the condition had on the patient's ability to undertake everyday activities e.g. driving, reading, hobbies, putting on make-up etc. These symptoms encompassed all the differing pathologies encountered as a result of nasolacrimal duct blockage; tear outflow obstruction, dacryocoele, mucocoele, mucopyocoele, acute dacryocystitis and fistula.

Accordingly, the questionnaire was constructed to give a numerical score for varying degrees of severity of each of the eye-specific symptoms, asking about each eye separately, and another score for social impact. It was found most appropriate to ask the patient about these symptoms as they could recall them over the previous eight weeks. In practice, when performing the questionnaire in clinic, it was found to be easy and quick to administer, taking less than 5 minutes per patient. The numerical score of the test for each patient (the 'Lac-Q score') is the sum of three scores (maximum score of 33): a symptom score for the right eye; a symptom score for the left eye (maximum possible score for each eye $=14$ ); and a score for social impact (maximum possible score $=5$ ).

\section{Study groups and clinical cases}

The main study group comprised 17 patients diagnosed with primary acquired nasolacrimal duct obstruction (NDO). Five patients underwent bilateral surgery, so there were 22 DCR operations performed in this group. The majority of the group were female $(n=11)$ and the mean age was 66 years. The pathologies encountered at operation were as follows: NDO alone $=10$ cases; $\mathrm{NDO}$ with non-purulent mucocoele $=8$ cases; NDO with infection (lacrimal mucopyocoele, or pus found in sac) $=4$ cases. For the separate study group of 12 preoperative patients in whom the questionnaire was re-administered to evaluate test-retest reliability, the mean age was 65 years. There were no incomplete questionnaires or missing clinical data in either group.

\section{Patient satisfaction and surgical success}

Following surgery, at the time of follow-up, 15 patients were satisfied with the result of the operation, while two were dissatisfied and requested revision. The results of postoperative clinical testing (stomal patency and FEDT test) for the 22 operations are summarised in Table 1.

Of the 15 patients who were satisfied following surgery, there was one patient in whom the stoma was found not to be patent and the FEDT showed no dye transit at 10 minutes. In this case, the patient reported a subjective improvement in symptoms as demonstrated by a decrease in eye symptom scores from 5 to 1 and social impact score from 4 to 1 . For the two patients who were not satisfied following surgery, objective evidence of surgical failure was demonstrated by stomal stenosis and no transit of fluorescein during the FEDT. There was also no significant reduction in their overall Lac-Q and individual domain scores postoperatively. Both patients accepted an offer to undergo revision surgery. Following revision, they were satisfied with the final result; the stoma was patent in both cases and transmitted fluorescein eye drops in 2- 5 minutes. Final post-revision Lac-Q 
A

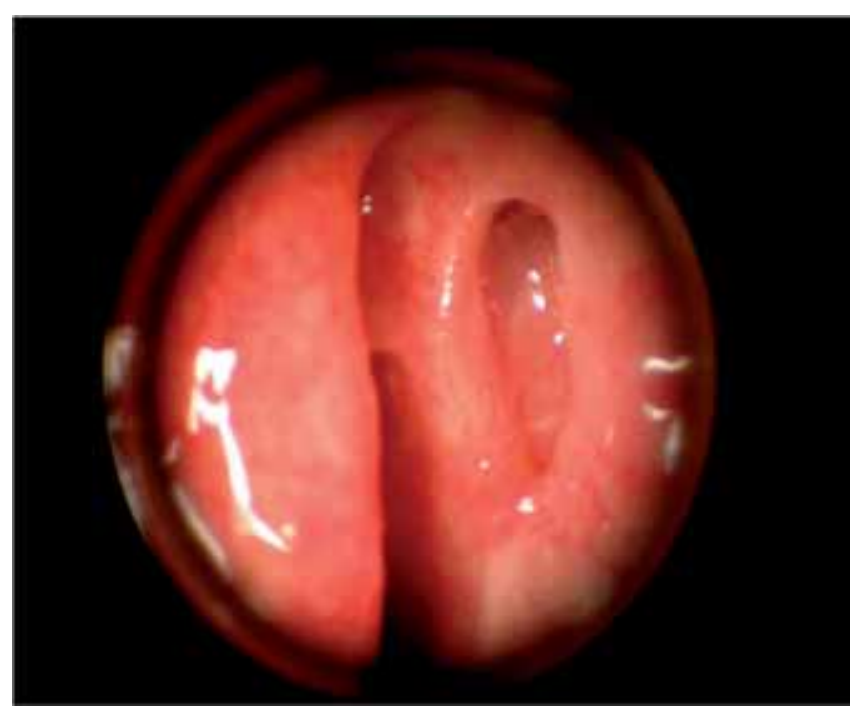

B

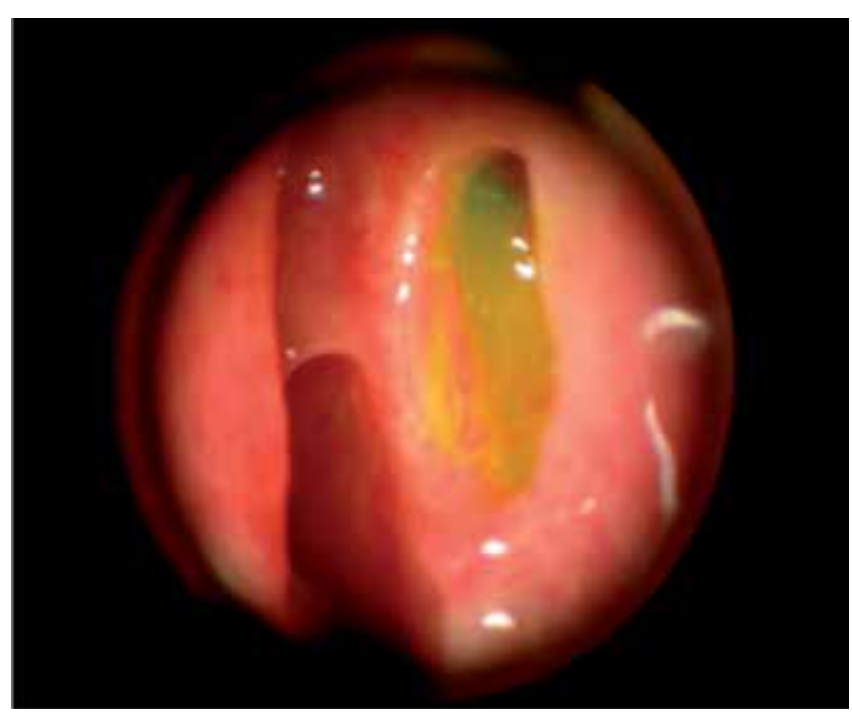

Figure 1. The functional endoscopic dye test (FEDT). (A) Endoscopic view of a left DCR stoma. A fluorescein eye drop is transmitted from the conjunctival sac to the stoma (B), confirming good lacrimal drainage function.

\section{Lac-Q Symptom Score}

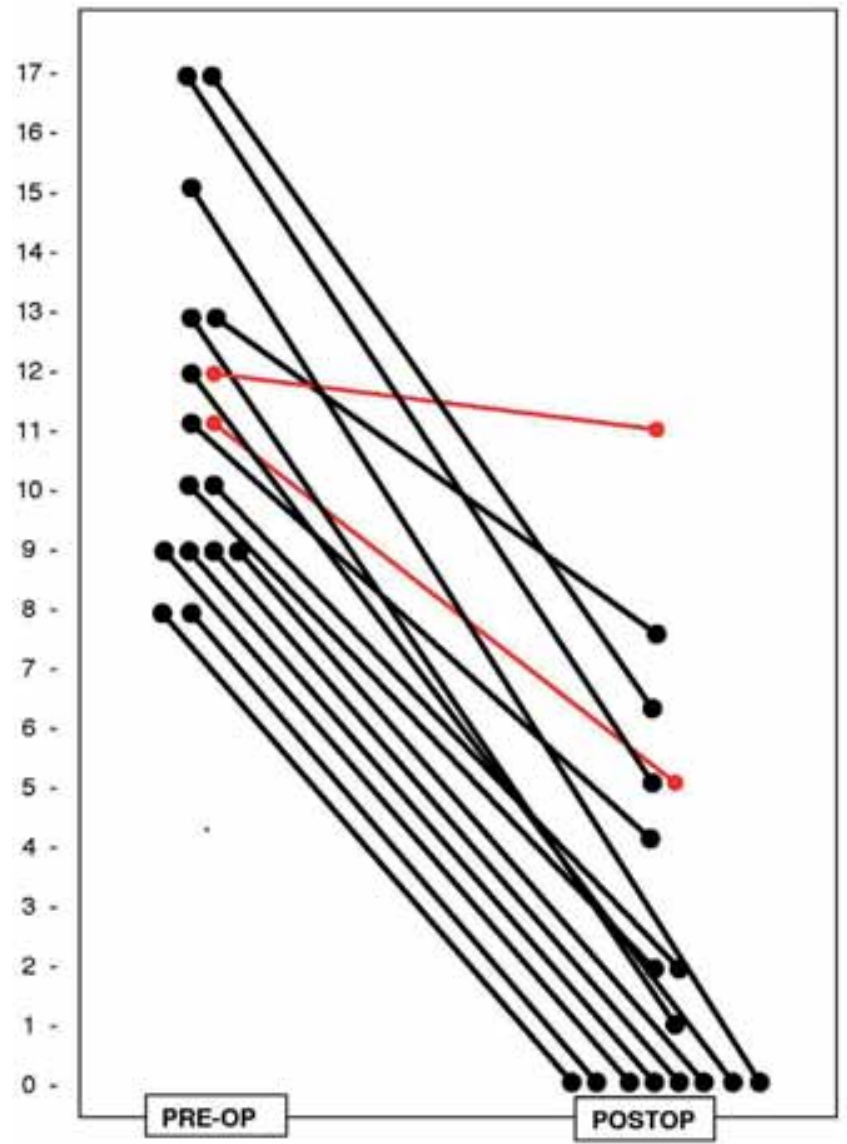

Figure 2. Preop and postop Lac-Q symptom scores for 17 patients. Black lines represent 15 patients satisfied with the outcome of surgery. Red lines represent the 2 patients who were not satisfied with the outcome of surgery. scores were 6 for one patient and 1 for the other.

The overall Lac-Q scores before and after surgery for the 17 patients are shown in Figure 2. It may be seen that pre-operatively, all patients scored at least 8 (out of a possible maximum of 33). After surgery, scores reduced to 8 or less in the 'satisfied' patients; satisfaction with surgery was associated with a reduction in score of at least 5 .

When analysing scores within the different domains of the questionnaire, it was also noted that pre-operative individual eye symptom scores were generally 3 or less (range $0-3$, median 1) for 13 non-troublesome eyes and 4 or above (range 4 - 10, median 6) for the 21 symptomatic eyes causing problems. This difference was found to be statistically significant $(p=0.002)$. The discrepancy between the number of symptomatic eyes and the number of operated eyes is explained by one patient who presented with symptoms in only one eye, but was noted to have bilateral mucocoeles in clinic and underwent bilateral surgery. Patients with unilateral eye symptoms consistently scored the symptomatic eye higher than the asymptomatic eye $(\mathrm{p}=0.001)$.

\section{Statistical analysis and validation of the questionnaire $\underline{1 .}$ Reliability of the questionnaire}

To test the questionnaire for internal consistency, eye symptom scores were compared to social impact scores. Significant correlation between the two measured variables was noted with Spearman's rank correlation coefficient $\left(r_{s}\right)$ of $0.8(p<0.001)$. Stability was assessed by analysing test-retest reliability within the group of 12 preoperative patients retested after a 4-8 week interval. Calculations showed excellent correlation between total Lac-Q scores and also when comparing individual eyesymptom and social impact scores $\left(r_{s} \geq 0.8\right)$ demonstrating high reliability of repeated measures. 


\section{Validity of the questionnaire}

The validity of the Lac-Q score in assessing outcome was tested by comparing postoperative scores with other outcome measures. Postoperative scores correlated well with FEDT results $\left(r_{s} \geq 0.7, p=0.002\right)$. Patients who expressed satisfaction with the procedure had lower postoperative Lac-Q scores than patients who were dissatisfied; again, this correlation was significant $(\mathrm{p}<0.05)$.

Assessment of correlation between pre-operative Lac-Q scores and severity of pathology was also undertaken. A definite trend between higher pre-operative symptom scores and more severe pathology was demonstrated, although correlation was weak $\left(r_{\mathrm{s}}=0.4, \mathrm{p}=0.04\right)$.

\section{Responsiveness to change}

When compared to pre-operative scores, the reduction in Lac-Q scores postoperatively was significant $(p=0.0002)$.

Similar results were found when looking within the questionnaire at the separate domains of questions. Post-operative eye symptom scores were assessed in all 22 operated eyes. It was found that there was a significant reduction in eye scores following surgery (median 6 vs. $0.5, p=0.0001$ ). This was also the case when comparing social impact scores, which also decreased significantly post-surgery (median 4 vs. $0, \mathrm{p}=$ 0.0007).

\section{DISCUSSION}

Improving patient symptoms and quality of life are key objectives when planning rhinological surgery. It is through the use of PROMs that the full symptomatic impact of intervention can be assessed and they are rapidly becoming an important accessory to clinical measurement ${ }^{(2)}$. With regards to lacrimal surgery, development of a symptom-based questionnaire has been problematic in view of the variety of pathologies caused by NDO and the marked variation in symptoms and social impact of disease experienced by the patient.

Various studies have attempted to formulate questionnaires to assess lacrimal surgery outcomes. Ho et al. (7) assessed 65 patients six months after DCR surgery. They used a generic quality of life questionnaire designed for assessment of otolarygological interventions, the Glasgow Benefit Inventory (GBI), together with clinical assessment of stomal patency, FEDT and documentation of epiphora. The study showed significantly superior GBI scores in those patients who underwent successful DCR. Use of the GBI by Speilmann et al. ${ }^{(1)}$ to assess outcomes following endonasal DCR also showed positive health impact results in 92 patients who completed the questionnaire. The authors concluded that endoscopic DCR was an effective and appropriate alternative to the more conventional external approach.

Other investigators have used symptom-specific questionnaires. A variety of studies have assessed outcomes related to single symptoms such as epiphora. Mansour et al. ${ }^{(13)}$ assessed the long-term outcome of external DCR in a total of 83 patients. Postal questionnaires assessing pre- and postoperative severity of tearing were used. The results showed a significant reduction in scores postoperatively, with a subjective success rate of $89 \%$ at one year following surgery. Others have also documented outcomes based on patient satisfaction or resolution of epiphora ${ }^{(8-10)}$.

A few studies have attempted to evaluate a broader spectrum of symptoms and the impact of lacrimal pathology on quality of life. Cheung et al. ${ }^{(14)}$ looked at the impact of lacrimal symptoms on day-to-day activities and levels of embarrassment in patients undergoing DCR, both pre- and postoperatively. They showed that successful surgery correlated with a reduction in scores postoperatively, but that the only significant reduction in score was related to embarrassment. Bakri et al. ${ }^{(12)}$ used the GBI in combination with 5 ocular-specific symptom questions (tear production, pus discharge, orbital swelling, orbital pain and change in vision) to assess outcomes following laser endonasal DCR (204 patients) and external DCR (78 patients). Again, following successful DCR, GBI scores showed positive changes in health status. The ocular-specific questions showed better outcomes only in those undergoing external DCR. Despite attempts to address lacrimal-specific questions, the authors only assessed postoperative outcomes and did not perform the questionnaire pre-operatively. Comparative analysis of improvement following the procedure could not, therefore, be carried out.

None of these studies fully addresses the difficulties outlined above in assessing symptoms in patients undergoing lacrimal surgery. Confining outcome measures to one main symptom (usually epiphora) may hide significant gains in other symptoms (e.g. pain), which may be of more importance to the individual patient undergoing surgery. The use of generic 'quality of life' questionnaires may be of limited use in a condition which often causes little disability ${ }^{(15)}$. The GBI is a quality of life tool, which usefully measures change in health status, is patient-orientated and designed to be maximally sensitive to otorhinolaryngological interventions. However, it is intended for post-procedure use ${ }^{(16)}$ and is therefore not appropriate for assessing severity of symptoms before surgery.

In this study, the Lac-Q questionnaire appears to be satisfactory in addressing these difficulties. It takes into account both eye-specific symptoms and social impact of disease, and yields a numerical score which has a simple clinical interpretability. Symptomatic patients wishing surgery had scores of at least 8 . Successful surgery was associated with a reduction of score of at least 5 (minimally important difference). Fifteen of the 17 patients had scores that achieved a 5 point or more reduction following surgery. The two in whom this wasn't the case were also the patients who requested revision surgery. This is an important measure as it demonstrates that the data generated by the questionnaire is meaningful and enables the clinician to make a rapid interpretation of various factors including poten- 
Table 1. Post-operative outcome measure results.

\begin{tabular}{|c|c|c|c|c|c|c|c|}
\hline & & \multicolumn{2}{|c|}{ Stomal Patency } & \multicolumn{4}{|c|}{ Functional endoscopic dye test (minutes) } \\
\hline & & Yes & No & $<2$ & $2-5$ & $5-10$ & $\begin{array}{c}>10 \\
\text { (No dye) }\end{array}$ \\
\hline \multirow{2}{*}{$\begin{array}{l}\text { Patient } \\
\text { Satisfaction } \\
\text { (n= no. of operated eyes) }\end{array}$} & $\begin{array}{l}\text { Yes } \\
(n=20)\end{array}$ & 19 & 1 & 16 & 3 & 0 & 1 \\
\hline & $\begin{array}{l}\text { No } \\
(n=2)\end{array}$ & 0 & 2 & 0 & 0 & 0 & 2 \\
\hline
\end{tabular}

tial outcomes based on the scores generated. Although it may be useful in guiding decision-making, it should not be used to exclude patients from surgery, as it uses a group average to establish what an important change is for an individual ${ }^{(3)}$.

Statistical tests of significance confirm the questionnaire to have content validity, in that the eye-specific symptoms correlate with the social impact scores. That is to say, the severity of eye symptoms is indeed the problem that causes social embarrassment, disability and clinic attendance for the patient. We attempted to address construct validity (validity of the actual content of the questions) by basing the questions on the reported experience of patients attending a rhinology clinic. This appears to have been a realistic approach, as evidenced by several findings in this study. For patients with unilateral disease, the patients' eye symptom scores were shown to be consistently higher in the troublesome eye, thus demonstrating discriminant validity. Furthermore, scores tended to be higher with increasing severity of lacrimal pathology. Postoperatively, lower scores were associated with patient satisfaction.

The Lac-Q score appears to respond appropriately to clinical intervention. Evaluation of test-retest variability show the questionnaire scores to be stable on repeat testing, confirming reliability in the absence of clinical change.

As discussed earlier, the terms "success" and 'failure' are difficult to apply definitively in lacrimal surgery. In this study, we have prospectively used several different measures (patient satisfaction, stomal appearance and functional endoscopic dye testing) to get as comprehensive an assessment of outcome as possible. Having said this, it is apparent that there is not always complete agreement between subjective and objective measures. This was evidenced in our study by the fact that one patient with objective stomal failure described her symptoms to be sufficiently improved as to consider the surgery a success, with a preoperative Lac-Q symptom score of 10 dropping to 2 after surgery. In addition, it may be seen in Figure 2, that both the patients who were dissatisfied with their surgery, and in whom stomal failure occurred, also noted some improvement in their symptom scores. It is interesting to speculate why this might be so. For any subjective measure, psychological factors such as cognitive dissonance or the placebo effect may influence symptom reporting. However, the correlation between eye scores and social impact remains good in both successes and failures, suggesting these psychological factors may not be so important in our study. A possible physiological explanation for this phenomenon may be that the surgery temporarily alters the function of the sac in tear absorption. It can be imagined that having infection and calculus removed at operation, and silastic tubes in place for a few months giving temporary drainage, actually reduces the inflammation and mucus-secreting elements in the previously infected / obstructed system. Hence, even if the stoma stenoses completely after the stenting tubes have been removed, the lacrimal function isn't as bad as before surgery; the non-infected sac, with less inflammation and secreting less mucus, can function better in absorbing tears than an infected one. Hence, the patient is less symptomatic than before the operation. However, if this is true, one might expect that eventually, infection will recur and the patient will become more symptomatic again.

The postoperative assessment at 8 weeks after stenting tube removal was chosen in this study because by that time, any postoperative inflammation, granulations and crusting had all settled and the stoma was easy to assess endoscopically. Long-term stomal failure after lacrimal surgery has been reported to occur up to 24 months ${ }^{(17)}$ and even as late as 97 months postoperatively ${ }^{(18)}$. The main consideration for the purposes of this study was not the long-term success of the procedure, but the relation between postoperative symptoms and stomal appearance.

A difficulty encountered when undertaking this study was assessing what Lac-Q scores could be expected for a nonsymptomatic 'healthy' population. The reason for this is that it is difficult to define 'healthy' when assessing NDO. As discussed above, it is not uncommon to have demonstrable NDO but minimal symptoms of disease or even be asymptomatic, as illustrated in our study by one patient who presented with unilateral symptoms but was found to have bilateral lacrimal mucocoeles. Furthermore, NDO is often partial; significant symptoms can occur before complete blockage occurs, leading to the clinical phenomenon of "functional" blockage despite patency to syringing tests ${ }^{(19)}$. In this study, we addressed this difficulty by using the symptom scores from the nontroublesome eye in the unilateral cases to derive data for what eye-specific scores could be expected in a healthy population. Using these results, we found that there was no overlap between the range of 'symptomatic' and 'non-symptomatic' eye scores. Although the overall study population is small, the results show that the performance of the Lac-Q is very encour- 
aging. A larger population study could therefore, form the basis of further work in this area, providing additional support and validation.

Symptoms secondary to lacrimal pathology are not trivial and can have a significant impact on patients' lifestyle. The findings of this study suggest that the Lac-Q is a brief questionnaire, which can be used by clinicians to capture the salient symptoms of lacrimal obstruction together with their impact on lifestyle. It can discriminate between levels of severity and detect changes with intervention. It can thus serve as a useful adjunct to clinical diagnosis and decision-making.

\section{CONCLUSIONS}

The Lac-Q questionnaire is a symptom-based questionnaire that has been developed to aid the surgeon and patient in assessing symptoms before and after DCR surgery. It is easy to administer and yields a numerical score that has a simple clinical interpretability. It can be used to monitor success or failure of treatment interventions. In this study, we have demonstrated its reliability, validity, and stability. It therefore appears to be a promising tool for use in patient-reported outcome measures.

\section{ACKNOWLEDGEMENTS}

We are very grateful to $\mathrm{Mr}$ Tristan Reuser for advice in the development of the questionnaire.

\section{AUTHORSHIP CONTRIBUTION}

NM: Questionnaire design and execution, data collection, review of literature, preparation of manuscript, analysis of results, statistical analysis, interpretation of results, discussion and conclusion. TJR: Generation of concept for study, questionnaire design and execution, data collection, surgeon performing DCR surgery, review of literature, preparation of manuscript, interpretation of results, discussion and conclusion. TR: Guidance with statistical analysis. CH: Statistical analysis, advice and guidance in the preparation of the manuscript

\section{CONFLICT OF INTEREST}

None

\section{REFERENCES}

1. Timmins N. NHS goes to the PROMS. BMJ. 2008; 336: 14641465.

2. Hopkins C. Patient reported outcome measures in rhinology. Rhinology. 2009; 47: 10-17.

3. Hopkins C, Gillett S, Slack R, Lund VJ, Browne JP. Psychometric validity of the 22-item Sinonasal Outcome Test. Clin.Otolaryngol. 2009; 34: 447-454.

4. Olver J. Causes of a watering eye. Colour atlas of lacrimal surgery, pp 30-7. Oxford: Butterworth Heinemann, 2002.

5. Hurwitz JJ. Physiology of the lacrimal drainage system. In
Hurwitz JJ, ed. The Lacrimal System, pp 23-8. Philadelphia: Lippincott-Raven Publishers, 1996.

6. Olver J. Adult lacrimal surgery. Colour atlas of lacrimal surgery, pp 131-42. Oxford: Butterworth Heinemann, 2002.

7. Ho A, Sachidananda R, Carrie S, Neoh C. Quality of life assessment after non-laser endonasal dacryocystorhinostomy. Clin. Otolaryngol. 2006; 31: 399-403.

8. Mathew MR, McGuiness R, Webb LA, Murray SB, Esakowitz L. Patient satisfaction in our initial experience with endonasal endoscopic non-laser dacryocystorhinostomy. Orbit. 2004; 23: 77-85.

9. Moore WM, Bentley CR, Olver JM. Functional and anatomic results after two types of endoscopic endonasal dacryocystorhinostomy: surgical and holmium laser. Ophthalmology. 2002; 109: 1575-1582.

10. Tripathi A, Lesser TH, O’Donnell NP, White S. Local anaesthetic endonasal endoscopic laser dacryocystorhinostomy: analysis of patients' acceptability and various factors affecting the success of this procedure. Eye (Lond) 2002; 16: 146-149.

11. Spielmann PM, Hathorn I, Ahsan F, Cain AJ, White PS. The impact of endonasal dacryocystorhinostomy (DCR), on patient health status as assessed by the Glasgow benefit inventory. Rhinology. 2009; 47: 48-50.

12. Bakri SJ, Carney AS, Robinson K, Jones NS, Downes RN. Quality of life outcomes following dacryocystorhinostomy: external and endonasal laser techniques compared. Orbit. 1999; 18: 83-88.

13. Mansour K, Sere M, Oey AG, Bruin KJ, Blanksma LJ. Longterm patient satisfaction of external dacryocystorhinostomy. Ophthalmologica. 2005; 219: 97-100.

14. Cheung LM, Francis IC, Stapleton F, Wilcsek G. Symptom assessment in patients with functional and primary acquired nasolacrimal duct obstruction before and after successful dacryocystorhinostomy surgery: a prospective study. Br J Ophthalmol. 2007; 91: 1671-1674.

15. Patrick DL, Deyo RA. Generic and disease-specific measures in assessing health status and quality of life. Med.Care 1989; 27: S217-S232.

16. Robinson K, Gatehouse S, Browning GG. Measuring patient benefit from otorhinolaryngological surgery and therapy. Ann.Otol. Rhinol.Laryngol. 1996; 105: 415-422.

17. Warren JF, Seiff SR, Kavanagh MC. Long-term results of external dacryocystorhinostomy. Ophthalmic Surg Lasers Imaging. 2005; 36: 446-450.

18. McMurray CJ, McNab AA, Selva D. Late failure of dacryocystorhinostomy. Ophthal Plast Reconstr Surg. 2011; 27: 99-101.

19. Olver J. Lacrimal assessment. Colour Atlas of Lacrimal Surgery, p 32. Oxford: Butterworth-Heinemann, 2002.

Miss Nina Mistry

Department of Otorhinolaryngology

Queen's Hospital

Burton-Upon-Trent

Staffordshire

United Kingdom

address: 201 The Broadway

Perry Barr

Birmingham B20 3EU

England, United Kingdom

Tel: +44-7739-205 729 
Appendix. The Lac-Q questionnaire.

\section{Lac-Q - The Lacrimal Symptom Questionnaire Name: Number: Date:}

\section{Social and lifestyle impact of tear duct problem}

Which of these five statements is true about the tear duct problem overall in the last eight weeks?

Please tick the box next to any true statement.

- Friends or family have commented about the watery eye problem.

- The watery eye problem has caused embarrassment in company.

- The watery/sticky eye problem has interfered with everyday activity, for example (underline each that applies): Reading .. Driving . Wearing make-up Wearing glasses .. Hobbies Other activity (specify):

- The vision is sometimes blurred because of the watery / sticky eye problem.

- Medical attendance: visit to the family doctor's surgery, or the hospital eye clinic, beeause of tear duct problem.

(Scoring score one point for each box ticked, maximum score $=5$ )

Total score for social impact:

\section{Broblems witheacheye separatels}

For each of the four problems (watery eye, pain, sticky eye or swelling), put a tick in the box next to the statement which best describes the situation over the last eight weeks.

Use the left hand column for the left eye, and the right hand column for the right eye.

\section{-Watery eye}

No watery eye problem

The eye waters oceasionally, mainly outdoors

Troublesome watering of the eye, indoors and outdoors, some days

Troublesome watering of the eye most days

Troublesome watering of the eye every day

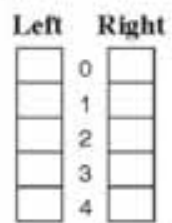

- Pain in or around the eye; soreness of eyelids

No pain

Some pain or soreness, but has not sought medical advice or treatment

Pain or soreness, has used prescription eyedrops

Painful and swollen (lacrimal abscess), requiring antibiotics or surgical drainage

- Sticky eye

No problem with sticky eye

The eye is sometimes sticky in the mornings

The eye is sticky every day in the mornings

The eye has sticky or mucous discharge throughout the day

There is infected discharge leaking through the skin of the lower eyelid (fistula)

-Swelling or lump at the medial canthus (mucocoele)

No swelling or lump

Swelling present, but only intermittently

Swelling present all the time

(Scoring: use numbers in central column)

Total scores for each eye:
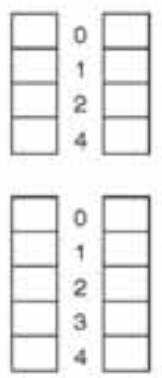

Lac-Q score (sum of three total scores):
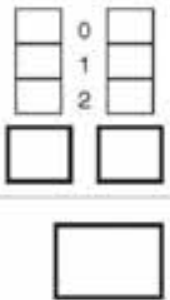

\section{ADVERTISEMENT}

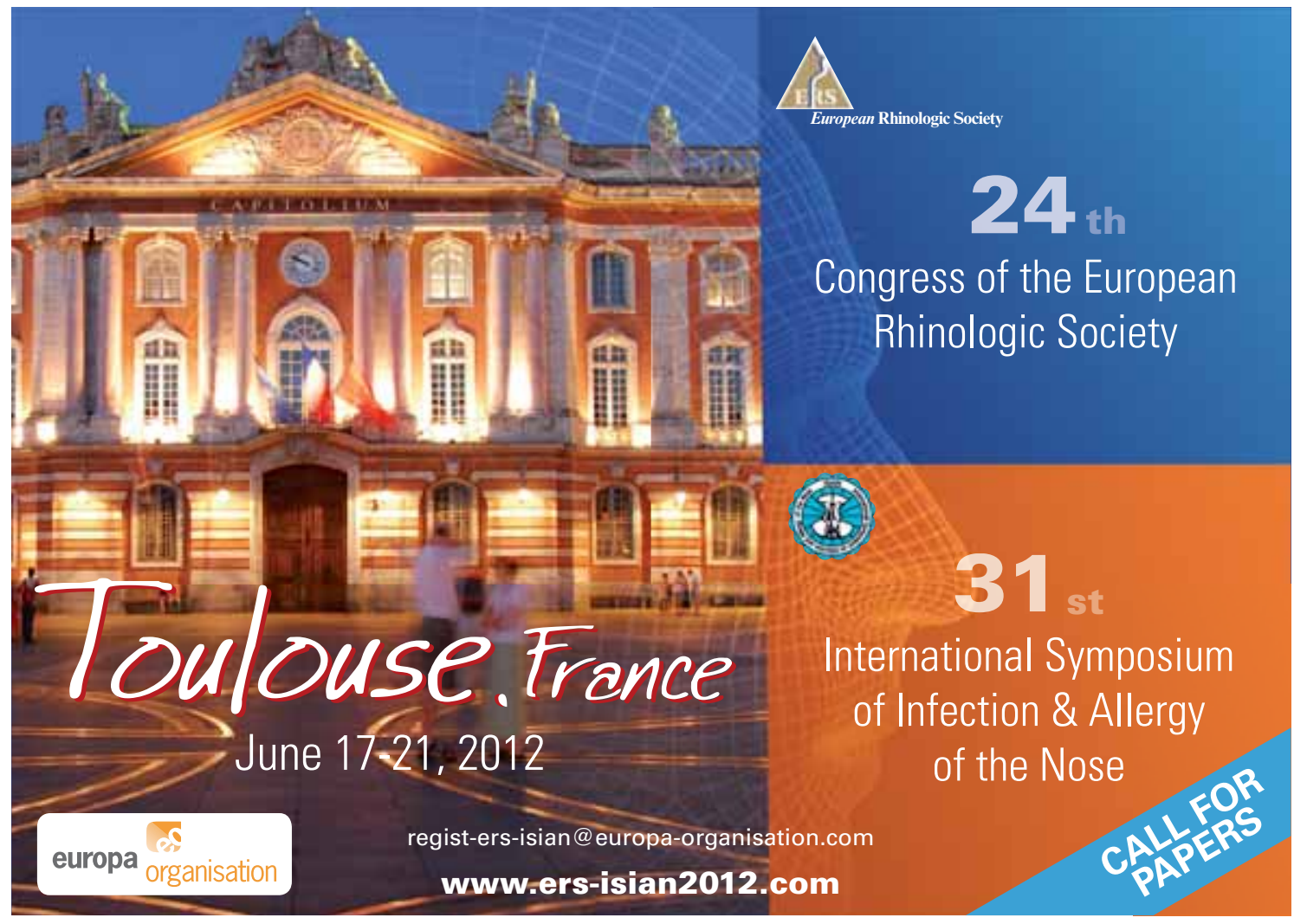

